\title{
A Study on the Dynamic Effect of Sports Promotion Strategy and Effectiveness of National Health Care in Taiwan
}

\author{
Tian-Syung Lan and Pin-Chang Chen \\ Department of Information Management, Yu Da University of Science and Technology, \\ Miaoli, Taiwan
}

\begin{abstract}
Based on system dynamics theory, this study used Vensim software to develop the Stock-Flow Diagram. This study used the Dynamic Situations of system dynamics to perform dynamic simulation on the effect of sports promotion strategy on Taiwan's national health care and its relevant medical expenditure and future expenditure. Moreover, this study used the simulation results as reference for strategy promotion, which can make it easier to promote strategies, reduce the pressure of increase in national health insurance and increase the success rate of policy implementation. The research results showed that, situational simulation of system dynamics can simulate the effect of development trend of effectiveness of Taiwan's national health care on the changes in complicated policy promotion system with time. The results of the simulation of policy situation showed that, it is necessary to adopt medium policy promotion and implementation rate of 55\% and national physical fitness increase rate of 5\% as the best strategy implementation rate in the future two years to obtain the greatest benefit.
\end{abstract}

\section{KEYWORDS}

System Dynamics, Sports Promotion Strategy, National Health Care

\section{INTRODUCTION}

The health of citizens is an important asset of a country, as well as the key factor for improving national competitiveness. High-quality human resources and high-efficiency productivity can only be created when citizens have healthy physique and plenty of thoughts to live a LOHAS life [1]. The foundation of national prosperity cannot be laid until the government promotes sports strategy to lead the wave of national sports and to further improve social economy [2,3]. Therefore, the cause-and-effect relationship between sports promotion strategy and effectiveness of national health care is an issue that cannot be ignored $[4,5]$.

This study mainly used the dynamic situational simulation of system dynamics to perform dynamic simulation on the relationship between sports promotion strategy and effectiveness of national health care in Taiwan and their future development. In addition, this study used the simulation results as the reference standards on amendment to sports promotion strategy $[6,7]$.

The purpose of this study is to use the dynamic situational simulation of system dynamics to find out the best sports promotion strategy to improve the effectiveness of national health care. Moreover, it is hoped that the promotion of sports strategy can lead the wave of national sports and strengthen citizens' awareness of improving health by taking exercise $[8,9,10,11]$. 


\section{SYSTEM DYNAMICS}

\subsection{System Dynamics Theory}

The concept of system dynamics originates from cybernetics, and system dynamics is a management tool that can assist managers in systemic thinking. In static studies, system will not change with the change of time. Therefore, it is impossible to analyse the complicated changes of systemic structure with the time. As a result, this study intends to investigate various mutual effects generated after the entire system changes with time to respond to external conditions based on the management concept of cause-and-effect feedback loops of system dynamics. Because system dynamics can effectively resolve the deficiency that variables cannot be easily quantified in general analytic measurement model and dynamic environment, the simulated system status can better reflect the actual situation of real environment [12].

\subsection{System Dynamics Model Development Procedures}

The founder of system dynamics, J.W. Forrester, suggested that the model development process includes the following six procedures [13]:

\subsubsection{Description on Current System Status}

The first step is to find out current urgent problems to be solved and expected objectives, and to describe the cause-and-effect relationships among system structure.

\subsubsection{Mathematical Model}

The second step is to convert the cause-and-effect relationships among the aforesaid system structure to a mathematical model of stock-flow equations.

\subsubsection{Simulation}

The third step is to use system dynamics software and simulation process to analyse whether the designed system and its cause-and-effect relationships are correct and reasonable. If they are not reasonable, the system will be amended until the correct and reasonable system model is developed.

\subsubsection{Design of Policy Structure}

The fourth step is to use system dynamics software to simulate various policies to further find out the best implementation policy.

\subsubsection{Education and Discussion}

The fifth step is to implement the educational training before the implementation of policy to enable participants to reach consensus and to ensure the success of policy implementation.

\subsubsection{Change in Policy Implementation Structure}

The last step is to completely exclude the concepts of the old policies, and to start to implement new policies and develop new information sources. 


\subsection{System Dynamics Model Development Criteria}

Sterman pointed out that model development process is highly innovative, and the model development approach of everyone is different. However, it is necessary to abide by the same rules for success [14].

\subsubsection{Clarify the Problems and Determine the Boundaries}

It is necessary to make sure the main variables affecting the system and their time unit and range.

\subsubsection{Form Dynamic Hypotheses}

It is necessary to propose dynamic hypotheses according to the problem. However, it is necessary to comply with endogenous perspectives to develop system cause-and-effect loop diagram.

\subsubsection{Develop System Dynamics Model}

It is necessary to define the parameters, structure and decision-making rules and to test the accuracy of hypotheses.

\subsubsection{Perform Tests Repeatedly}

It is necessary to constantly simulate and test the system structure to find out the model with the highest reliability and validity.

\subsubsection{Design and Assess Policies}

It is necessary to test the practicability of policy implementation in real environment based on the model as the reference information for managers to develop policies.

Sternman emphasized that the aforementioned model development process is not linear procedures, but a process of constant revisions. The new idea of any procedure will affect other procedures. The model development process of this study is compliant with this criterion.

\section{RESEARCH METHOD}

This study used Vensim software of system dynamics to allocate the proportions of the effect of high, medium and low sports policy implementation rate on the effectiveness of national health care, as well as to simulate the system dynamics situation affecting the future development [15]. Firstly, this study determined which variables should be stock, and then input relevant cause-andeffect variables into the stock-flow diagram one by one to develop the system dynamics model. The Causal Feedback Loop Diagram and Stock-Flow Diagram of this study are as follows:

\subsection{Causal Feedback Loop Diagram}

As shown in Figure 1, when the overall effectiveness of national preventive health care of a country is good, the number of patients attending clinic visits covered by national health insurance (NHI) will be decreased, namely, the medical care expenditure of a country will be reduced. In other words, when a country's medical care expenditure is less, the government can use adequate NHI funding. When the government has sufficient NHI funding, it can promote more sports policies thunder the situation of sufficient funding, the implementation rate of various 
policies will be increased relatively. When there is an increase in the implementation rate of sports policies that are beneficial to the health of citizens, the increase rate of national physical fitness will also be increased. The effectiveness of national preventive health care can be improved through the increase in national physical fitness.

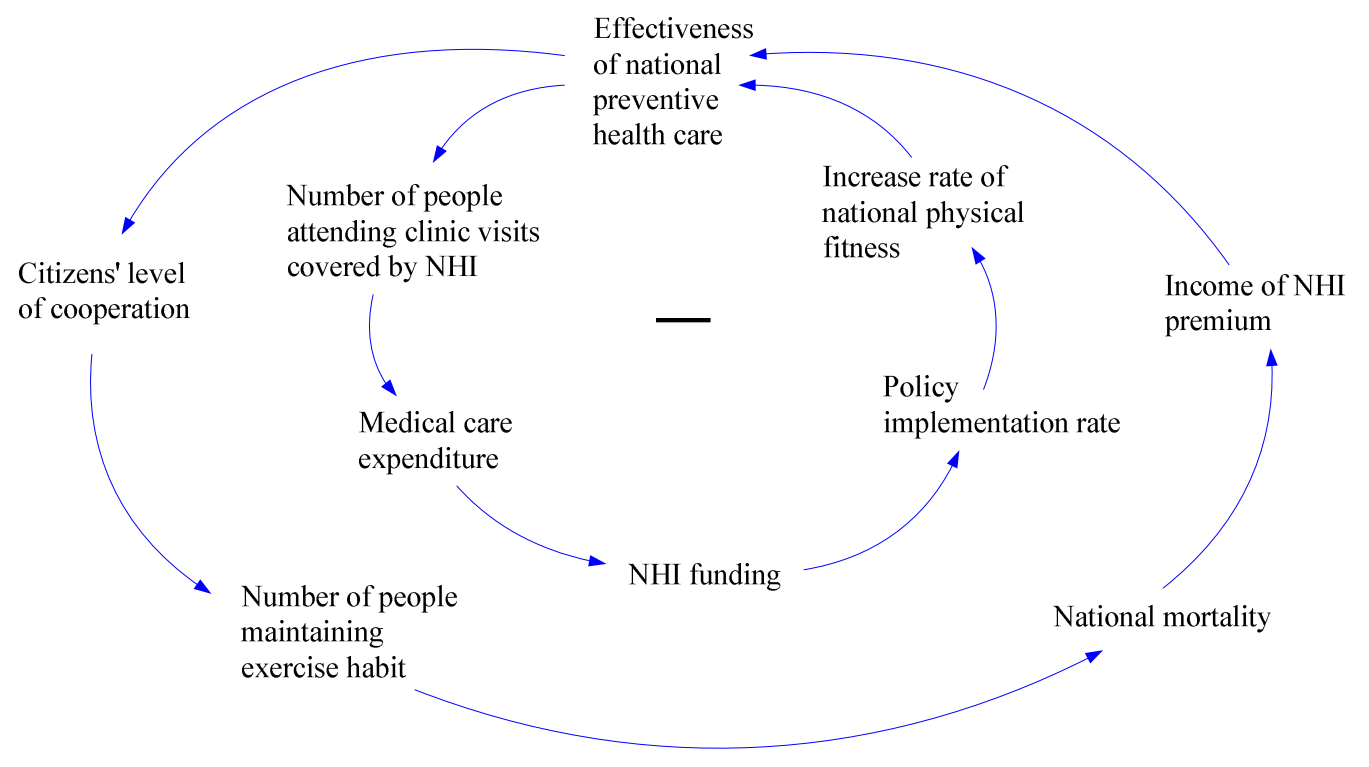

Figure 1. Causal Feedback Loop Diagram

Good effectiveness of national preventive health care also means that all the citizens are highly cooperative with the physical and psychological health development sports policies promoted by the government. The high level of cooperation further increased the number of people who constantly maintain exercise habit. Moreover, strong physique can be developed and the aging of various physical functions can be alleviated by improving national physical fitness. The alleviation of aging of physical functions also reduces national mortality. When the national mortality is reduced, there will be more citizens paying the NHI premium, which will significantly increase the income of NHI. In other words, the significant increase in the income of NHI means that the effectiveness of a country's national preventive health care is good.

\subsection{Stock-flow Diagram}

As shown in Figure 2, the model was firstly initiated using the variable of policy implementation rate. To promote the atmosphere of national sports and improve national physical fitness, the increase in the physical fitness can prevent the occurrence of diseases, reduce national mortality and increase the average life expectancy of citizens. The increase in life expectancy of citizens can lead to relative increase the number of citizens paying the NHI premiums and further reduce county/city governments' burden of debts to NHI. The higher the increase rate in national physical fitness is, the better the effectiveness of national preventive health care is, which also indirectly decreases the number of people attending clinic visits covered by the NHI. When the number of people attending clinic visits is decreased, the medical care expenditure will also be decreased, which enables the government to have more funding to cover the expenses of citizens' catastrophic illness and implement the policies to take care of the disadvantaged groups. 


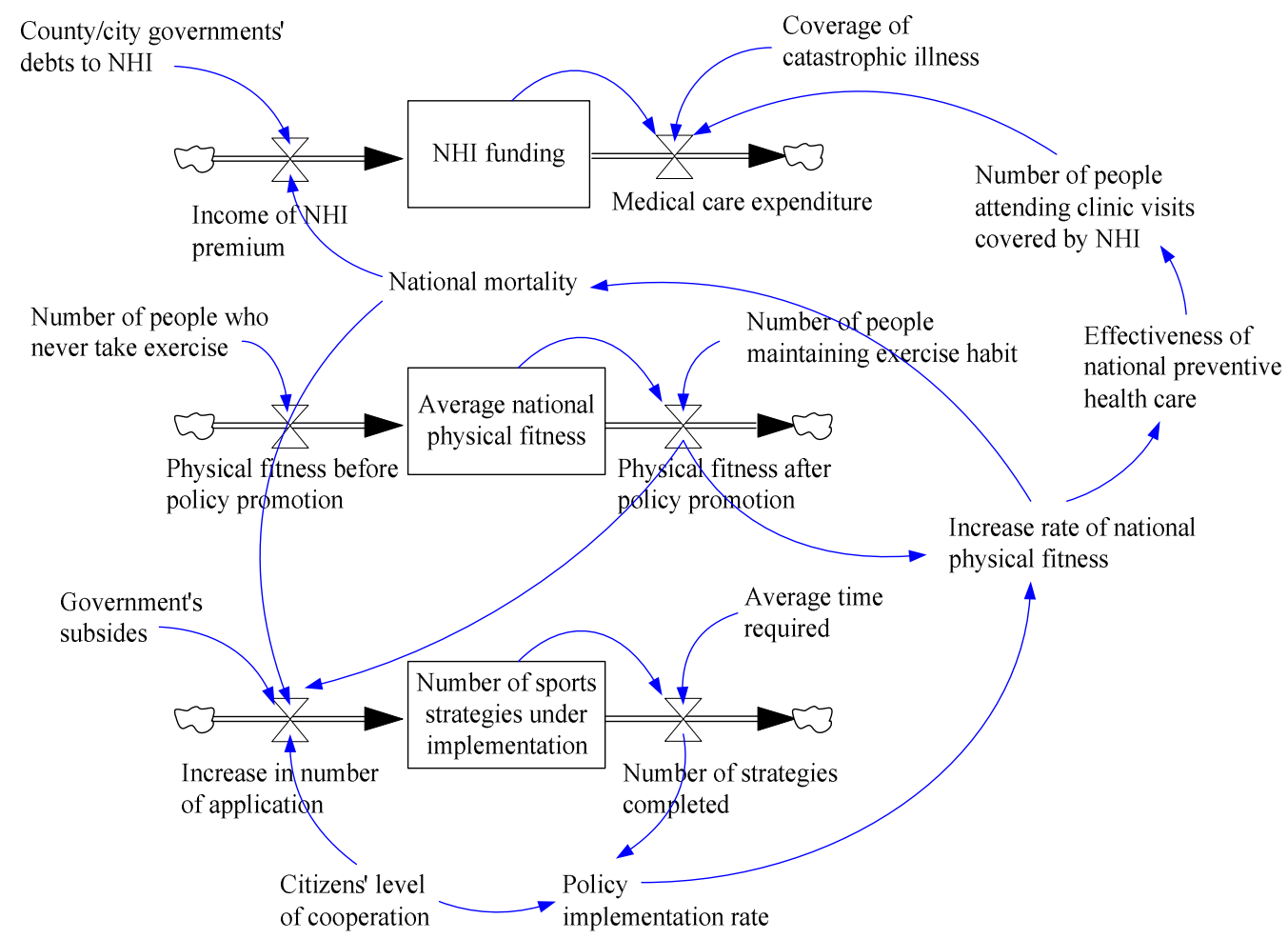

Figure 2. Stock-flow Diagram

If the national mortality cannot be reduced, the government has to spend more funding on increasing citizens' willingness to take exercise to make those who never take exercise to understand the importance of sports to physical health and to decrease the number of citizens who never take exercise. In addition, it can also increase the number of people who constantly take exercise to further achieve the dual effect. In short, when citizens are highly cooperative, the number of policies completely implemented will also be increased relatively to facilitate the implementation of the overall policy and to achieve the objective. Moreover, the people promoting policy can more aggressively implement the programs that are beneficial to the physical and psychological health of citizens. Therefore, the pressure of increase in NHI premiums can be alleviated to quell discontent of citizens and enhance their identification for government.

\section{RESULTS AND DISCUSSION}

This study chose to investigate the effect of high, medium and low policy implementation rate on effectiveness of national health care and its future development trend, and thus performed a 2year simulation test. Firstly, this study adjusted two variables, policy implementation rate and national mortality. Secondly, this study compared the conditions, NHI premium, medical care expenditure and effectiveness of national preventive health care to find out the policy promotion rate for the best effectiveness of national health care. 


\subsection{Strategy Situation 1}

This study set up the conditions: high policy implementation rate (85\%), national mortality rate $(20 \%)$, increase rate of national physical fitness of 5\%, citizens' level of cooperation (0.5) to perform simulation, as shown in Figure 3.

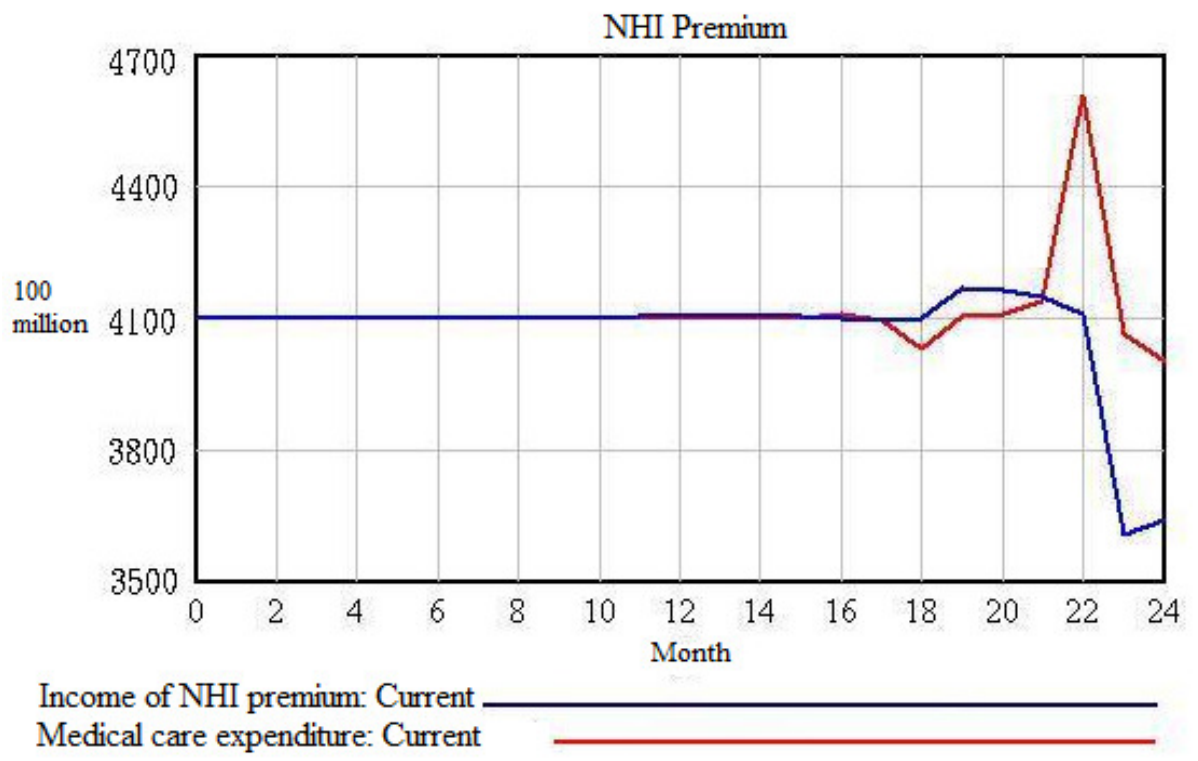

Figure 3. NHI Premium under High Policy Implementation Rate

When the government aggressively promoted national sports strategy with income of NHI premium and medical care expenditure equally (NTD 410 billion), the citizens engaged in sports in the first 11 months. Therefore, the physical fitness and immunity of most of the citizens were slightly improved, leading to the decrease in the number of people attending clinic visits. Therefore, the income of premium exceeded its expenditure. However, by Month 22, the expenditure (NTD 460 billion) significantly exceeded the income (NTD 410 billion), reflecting a deficit. The reason was that the physical and psychological fatigue caused by long-term excessive exercise led to and decrease in immunity. Therefore, the number of people attending clinic visits increased, and so did the expenditure.

As shown in Figure 4, under high policy implementation rate, the future development trend of effectiveness of national health care decreased from $20 \%$ in Month 15 before policy implementation to $16 \%$ in Month 18 in the midway. The reason was that, the effectiveness of policy implementation was not immediately reflected in the beginning, leading to citizens' misunderstand that the government was lying to them. However, by Month 21 when the effectiveness was significant, the development trend rapidly increased to $79 \%$ due to positive appraisal. However, the development trend slumped in the end, reflecting the short-lived development trend. The reason was that the government provided subsidies for participation in sports activities in order to promote national health care sports to the public, leading to a rush of participation in various health care sports. As a result, the excessive subsidies provided by the government led to the decrease and termination of health care sports strategy. 
International Journal of Computer Science \& Information Technology (IJCSIT) Vol 6, No 1, February 2014

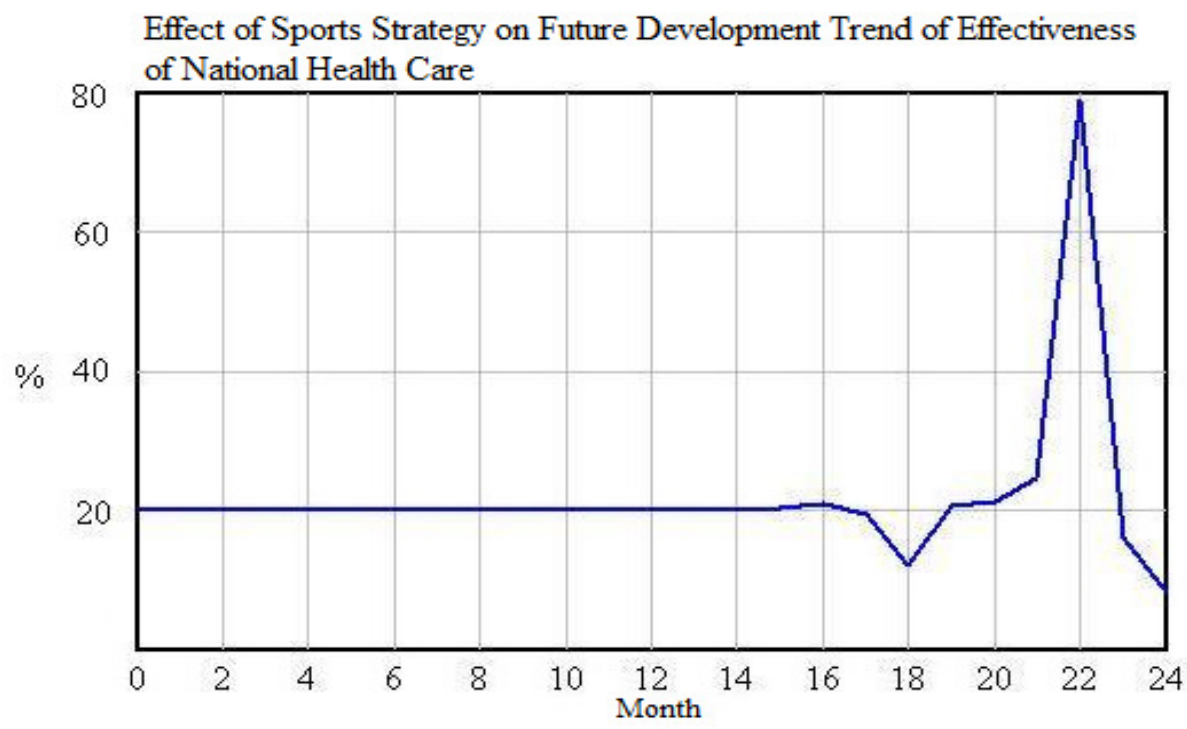

Effectiveness of National Preventive

Health Care: Current

Figure 4. Development Trend of Effectiveness of National Health Care under High Policy Implementation Rate

\subsection{Strategy Situation 2}

This study set up the conditions: medium policy implementation rate (55\%), national mortality $(20 \%)$, increase rate of national physical fitness of $5 \%$ and citizens' level of cooperation (0.5) to perform simulation, as shown in Figure 5.

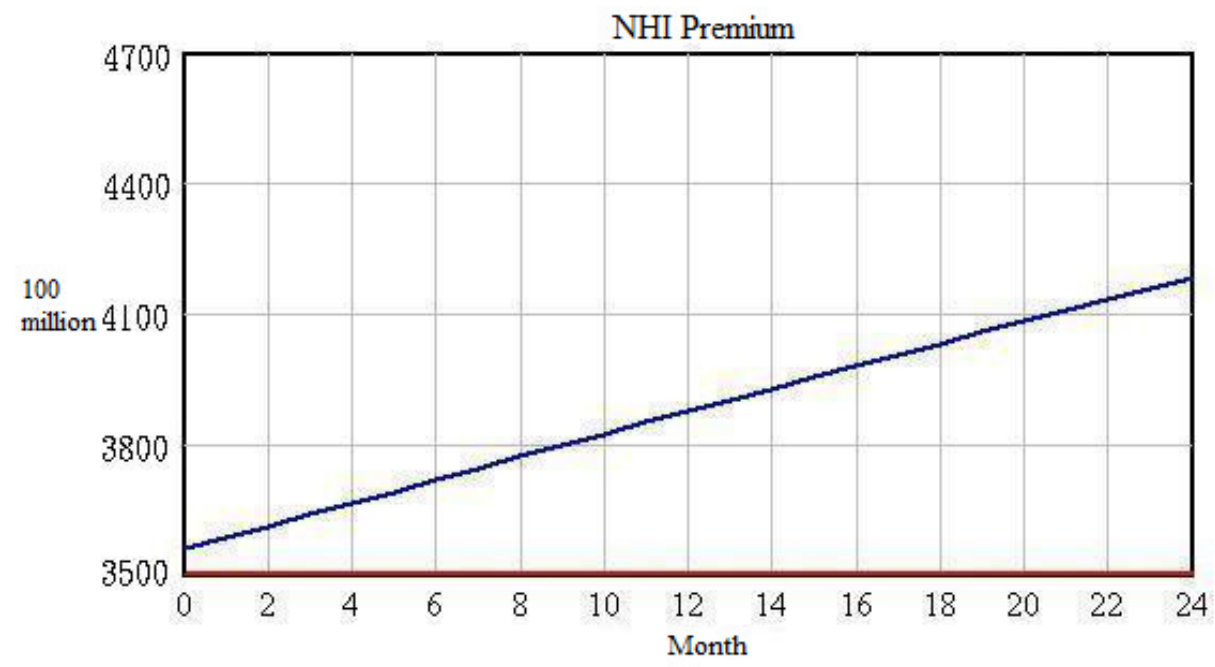

Income of NHI premium: Current Medical care expenditure: Current

Figure 5. NHI Premium under Medium Policy implementation Rate 
When the government properly promoted national health sports strategy, the income of premium gradually increased from NTD 360 billion in the beginning of implementation of policy to NTD 415 billion in the end. During this process, medical care expenditure was not increased. The reason was that this strategy was different from strategy 1 where citizens intended to obtain the subsidies from the government by participating in various health care sports activities held by the government, which led to deficit. This strategy was to properly provide subsidies according to the actual situation at that time. This approach would not lead to the decrease in the future funding, which might make citizens give up participation due to economic burden.

As shown in Figure 6, the future development trend of effectiveness of national health care increased from $4 \%$ in the beginning of policy implementation to $65 \%$ in the end. Although it was not as high as that that of strategy 1 (79\%), it could last longer in the future. The reason was that this strategy was different from strategy 1 where the government provided a lot of subsidies to promote national health sports strategy, leading to the short-lived development trend. This strategy was that the government provided subsidies in a timely manner to make the strategy last longer.

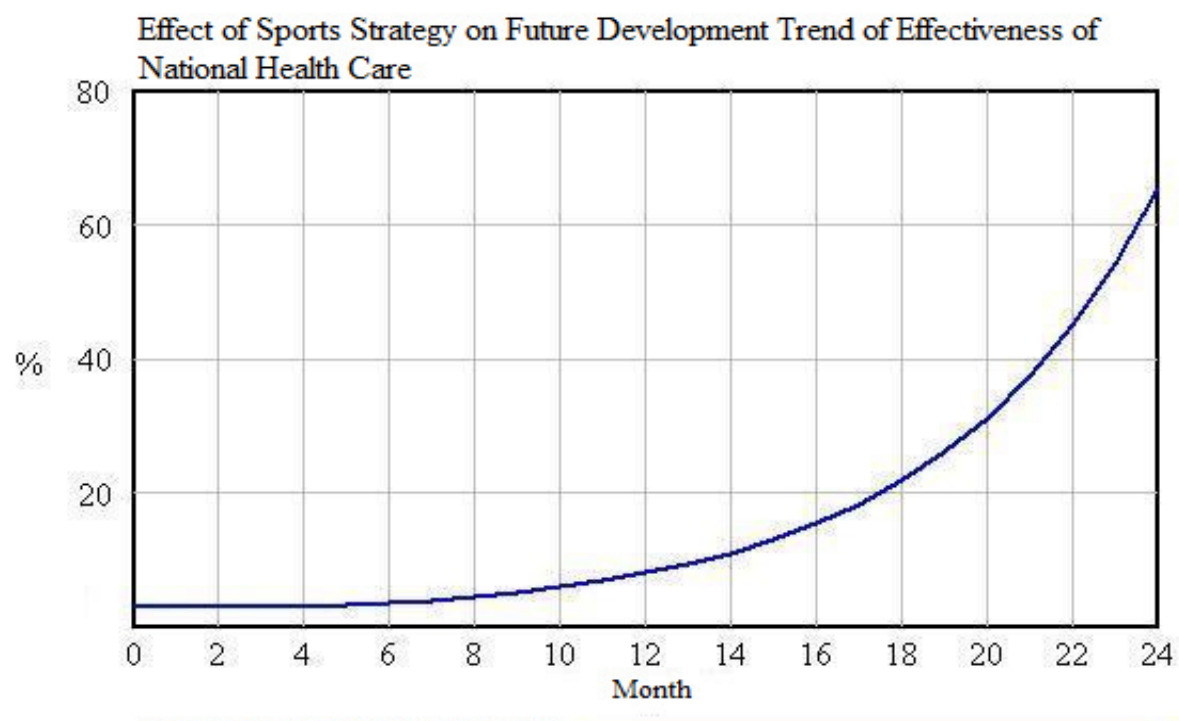

Effectiveness of National Health Care: Current

Figure 6. Development Trend of Effectiveness of National Health Care under Medium Policy Implementation Rate

\subsection{Strategy Situation 3}

This study set up the conditions: low policy implementation rate (25\%), national mortality (20\%), increase rate of national physical fitness of 5\% and citizens' level of cooperation (0.5), as shown in Figure 7. 


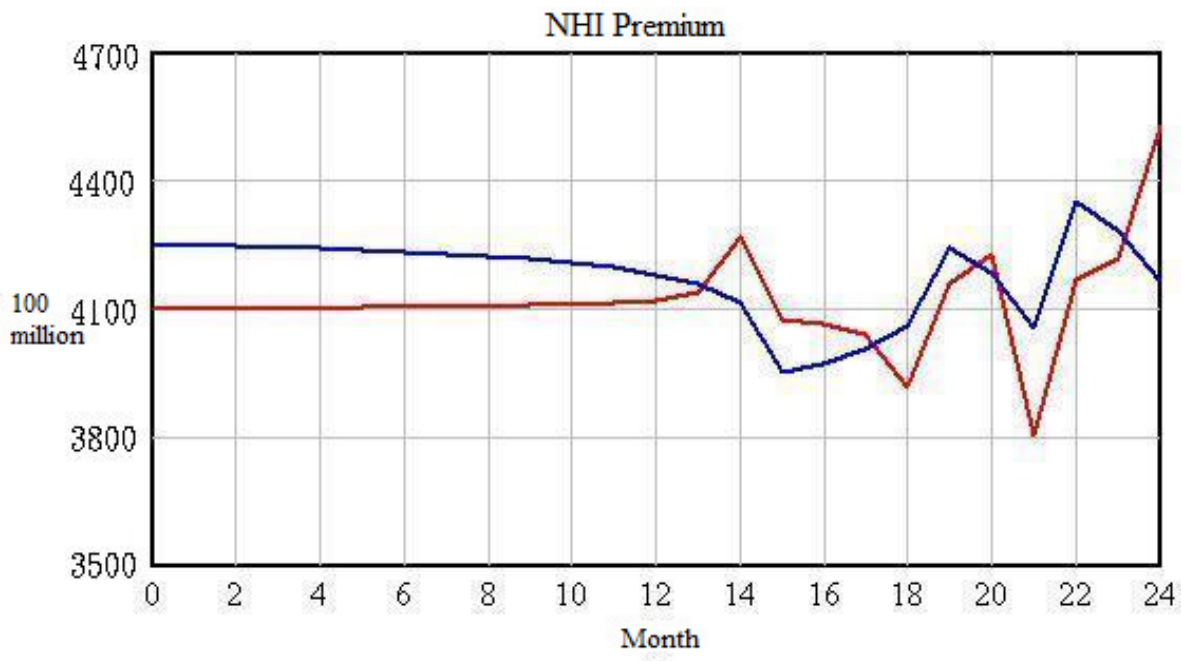

Income of NHI premium: Current-

Medical care expenditure: Current

Figure 7. NHI Premium under Low Policy Implementation Rate

When the government no longer attached importance to the promotion of national health care sports strategy, the original surplus of NHI premium of NTD 15 billion before the policy promotion decreased to NTD -35 billion (income of NHI premium of NTD 415 billion expenditure of NHI premium of NTD 450 billion) after a period of time. The reason was that the population structure in Taiwan had become an aging society. If citizens failed to alleviate the aging of their physical functions by taking exercise in their daily life to strengthen their immunity against diseases, the NHI premium would rise in the end to support the continuous implementation of NHI policy.

As shown in Figure 8, under low policy implementation rate, the future development trend of effectiveness of national health care decreased from $0 \%$ in Month 9 in the beginning to $-16 \%$ in Month 14. The reason was that the government did not aggressively promote the importance of sports to health and preventive health care. Moreover, because citizens were busy in their daily life, most of them neglected to maintain a good exercise habit. Although only a small proportion of citizens maintained exercise habit to contribute to the temporary growth of $25 \%$ in Month 21, the influence of real living environment still led to the slump of development trend of effectiveness of national preventive health care. 


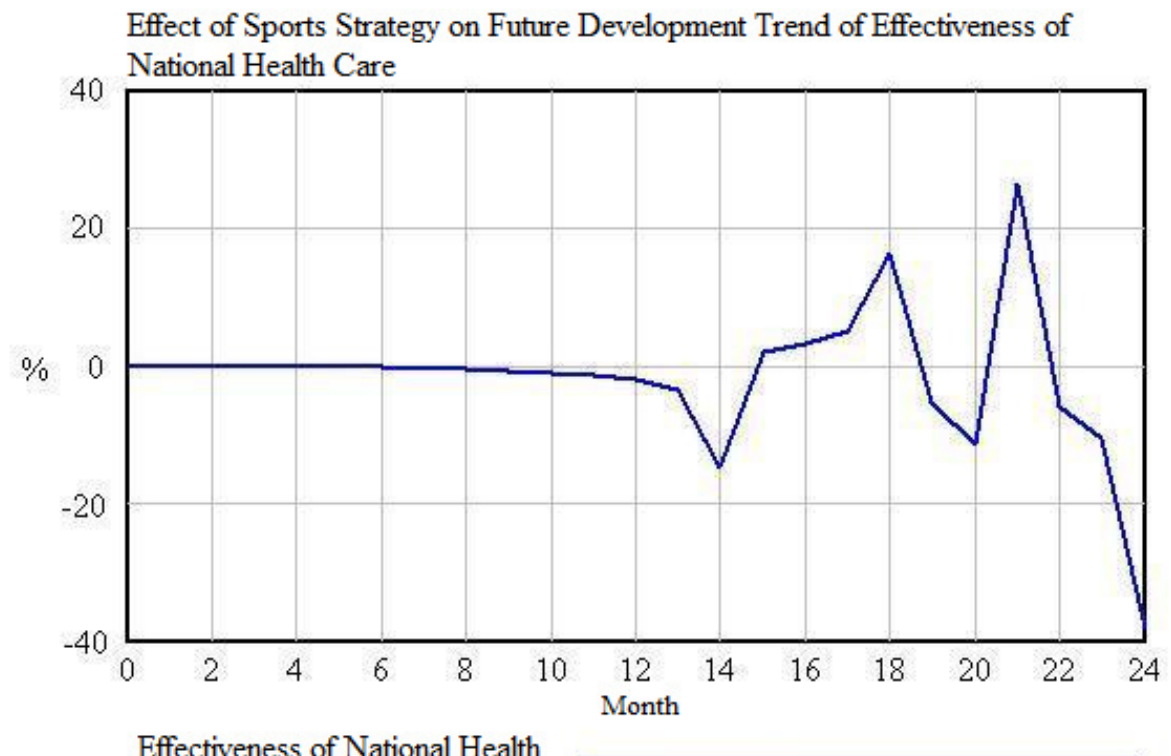

Care: Current

Figure 8. Development Trend of Effectiveness of National Heath Care under Low Policy Implementation Rate

The simulation results of the dynamic policy model in this study showed that, future policy makers may refer to relevant conditions of strategy situation 2 for the promotion rate and implementation rate of national health care sports strategy to develop, design and promote the detailed sports strategy. Moreover, in terms of subsidies, excessive subsidies will lead to increase in the number of application for implementation, as well as responsible unit's failure to engage in implementation. Insufficient subsidies will lead to poor implementation effectiveness and the eventual failure. Therefore, excessive and insufficient subsidies are both inappropriate. It is necessary to carefully assess and properly increase or decrease the subsidies according to the current status.

\section{CONCLUSION}

This study used system dynamics' advantages of ability to deal with a large number of variables and advanced nonlinear system, and performed dynamic simulation on strategy situation to choose the allocation of proportions of policy promotion with the best national health care effectiveness. According to research results, the government' level of importance attached to sports policy promotion, implementation rate and national mortality all have a significant effect on the future development of effectiveness of national health care, and may further directly affect the overall national strength. According to the simulation results of the strategy situation, medium policy promotion and implementation rate $(55 \%)$ and increase rate of national physical fitness of $5 \%$ should be adopted for the best effectiveness in the future two years.

However, the government usually aggressively promotes most of the strategies in the beginning, and then terminated them after a period of time due to lack of effectiveness. The government ignores the fact that it takes a very long time, instead of only a few months, for a promoted policy to achieve effectiveness. In Taiwan, no importance was attached to national health care sports in the past. It was not until many studies show that physical activities can prevent occurrence of 
cardiovascular diseases was the attention attracted to such an issue. If the promotion of policy can lead the wave of national sports, as well as encourage citizens to take exercise regularly to increase cardiopulmonary function and immunity to prevent diseases, a consideration amount of medical care expenditure can be saved for Taiwan, and the increase in NHI premium can be postponed. The results can be provided as reference for the government authority to plan for allocation of proportions in future policies, in order to further improve physical fitness of citizens in Taiwan, as well as to take the opportunity to strengthen national power.

\section{ACKNOWLEDGEMENTS}

Financial support of this work was provided by Sports Administration, Ministry of Education, Taiwan, (Project No. 102R-06-1-63).

\section{REFERENCES}

[1] Fullerton, S. \& Merz, G. R., (2008) "The four domains of sports marketing: a conceptual framework", Sport Marketing Quarterly, Vol. 17, No. 2, pp. 90-108.

[2] Berwick, D. M., (2003) "Disseminating innovations in health care", The Journal of the American Medical Association, Vol. 289, No. 15, pp. 1969-1975.

[3] Campinha-Bacote, J., (2002) "The process of cultural competence in the delivery of healthcare services: a model of care", Journal of Transcultural Nursing, Vol. 13, No. 3, pp. 181-184.

[4] Plsek, P. E. \& Greenhalgh, T., (2001) "The challenge of complexity in health care", British Medical Journal, Vol. 323, No. 7313, pp. 625-628.

[5] Mays, N. \& Pope, C., (2000) "Qualitative research in health care: assessing quality in qualitative research", British Medical Journal, Vol. 320, No. 7227, pp. 50-52.

[6] Ko, Y. J. \& Pastore, D. L., (2005) “A hierarchical model of service quality for the recreational sport industry”, Sport Marketing Quarterly, Vol. 14, No. 2, pp. 84-97.

[7] Chalip, L., (2006) "Toward a distinctive sport management discipline”, Journal of Sport Management, Vol. 20, No. 1, pp. 1-21.

[8] Dangerfield, B. C., (1999) "System dynamics applications to European health care issue", Journal of the Operational Research Society, Vol. 50, pp. 345-353.

[9] Coyle, G., (2000) "Qualitative and quantitative modelling in system dynamics; some research questions”, System Dynamics Review, Vol. 16, No. 3, pp. 225-244.

[10] Lyneis, J. M., Cooper, K. G. \& Els, S. A., (2001) "Strategic management of complex projects: a case study using system dynamics”, System Dynamics Review, Vol. 17, No. 3, pp. 237-260.

[11] Homer, J. B. \& Hirsch, G. B., (2006) "System dynamics modelling for public health: background and opportunities", American Journal of Public Health, Vol. 96, No. 3, pp. 452-458.

[12] Sterman, J. D., (2000) Business dynamics: Systems thinking and modelling for a complex world, Boston, USA: Irwin McGraw-Hill.

[13] Forrester, J. W., (1971) World dynamics, Cambridge, USA: The M.I.T. Press.

[14] Sterman, J. D., (2001) "System dynamics modelling: tools for learning in a complex world", California Management Review, Vol. 43, No. 4, pp. 8-25.

[15] Eberlein, R. L. \& Peterson, D. W., (1992) "Understanding models with Vensim”, European Journal of Operational Research, Vol. 59, No. 1, pp. 216-219. 